\title{
Free triiodothyronine/free thyroxine (FT3/FT4) ratio is strongly associated with insulin resistance in euthyroid and hypothyroid adults: a cross-sectional study
}

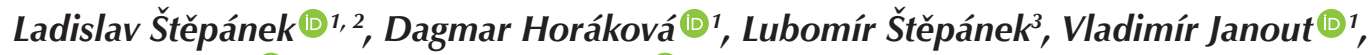 \\ Jana Janoutová(i) ${ }^{4}$, Kateřina Bouchalová ${ }^{5}{ }^{5}$, Karel Martiník ${ }^{6}$ \\ ${ }^{1}$ Department of Public Health, Faculty of Medicine and Dentistry, Palacký University Olomouc, Olomouc, Czech Republic \\ ${ }^{2}$ Department of Occupational Medicine, University Hospital Olomouc and Faculty of Medicine and Dentistry, \\ Palacký University Olomouc, Olomouc, Czech Republic \\ ${ }^{3}$ Institute of Biophysics and Informatics, First Faculty of Medicine, Charles University, Prague, Czech Republic \\ ${ }^{4}$ Department of Healthcare Management, Faculty of Health Sciences, Palacký University Olomouc, Olomouc, Czech Republic \\ ${ }^{5}$ Department of Pediatrics, University Hospital Olomouc and Faculty of Medicine and Dentistry, Palacký University Olomouc, \\ Olomouc, Czech Republic \\ ${ }^{6}$ Ambulance Prof. MUDr. Karla Martinika DrSc. s.r.o, Hradec Králové, Czech Republic
}

\begin{abstract}
Introduction: Insulin resistance (IR) is a key and early pathogenetic mechanism of cardiometabolic diseases with huge potential if detected early and mitigated, for lowering the burden of the disease. Available data are conflicting to what extent adult thyroid dysfunction is associated with IR. Therefore, we aimed to investigate the association and to identify which thyroid parameters are predictors of IR. Material and methods: After undergoing basic anthropometric and biochemical studies including thyroid hormones, oral glucose tolerance test (OGTT), and insulin, 1425 middle-aged individuals were divided into three groups according to thyroid parameters: overt hypothyroidism $(\mathrm{OH})$, subclinical hypothyroidism $(\mathrm{SH})$, and euthyroidism $(\mathrm{EU})$.

Results: The homeostasis model assessment of IR (HOMA-IR), fasting insulin, and two-hour glucose levels of OGTT showed a steady, yet insignificant, increase from EU through SH to OH. The strongest noted correlations were those of insulin levels with free triiodothyronine/ /free thyroxine (FT3/FT4) ratio $(r=0.206, \mathrm{p}<0.001)$ and FT3 $(\mathrm{r}=0.205, \mathrm{p}<0.001)$. Also in the case of HOMA-IR, the only statistically significant correlations were observed for FT3 $(r=0.181, p<0.001)$ and the FT3/FT4 ratio $(r=0.165, p<0.001)$. Among other thyroid hormones, linear logistic regression proved the FT3/FT4 ratio as the only significant predictor of HOMA-IR (linear coefficient $=5.26$, $p=0.027$ ) and insulin levels (linear coefficient $=18.01, p=0.023$ ), respectively. Thyroid-stimulating hormone was not associated with IR in either correlation or regression analysis.

Conclusions: The FT3/FT4 ratio should be more emphasised in the diagnosis and treatment of thyroid disorders. Patients could benefit from a pharmacological reduction of the FT3/FT4 ratio, potentially leading to a decrease in insulin resistance, and thus a corresponding decrease in the risk of the cardiometabolic diseases. (Endokrynol Pol 2021; 72 (1): 8-13)
\end{abstract}

Key words: thyroid disorder; insulin resistance; FT3/FT4 ratio; hypothyroidism; euthyroidism

\section{Introduction}

Thyroid disorders (TDs) are common conditions with potentially devastating health consequences that affect all populations worldwide. Both hypothyroidism and hyperthyroidism have their overt, clinically manifested forms caused by serum abnormities of the thyroid gland hormones (free thyroxine [FT4] and free triiodothyronine [FT3]). Whereas subclinical forms of TDs show normal serum levels of free thyroid hormones, there are inverse feedback changes in thyroid-stimulating hormone (TSH) levels [1-3].

The prevalence of overt hypothyroidism $(\mathrm{OH})$ in the general adult population varies between $0-2 \%$ and $5-3 \%$ in Europe and between $0-3 \%$ and $3-7 \%$ in the USA, depending on the definition used [1]. The diagnosis of subclinical hypothyroidism (SH) is biochemical, solely based on thyroid function testing. In iodine-sufficient populations, $\mathrm{SH}$ affects up to $10 \%$ of the population [2]. The prevalence of hyperthyroidism is $0.8 \%$ in Europe and $1.3 \%$ in the USA [3].

Proper thyroid function is important for the prevention of cardiometabolic diseases. The importance of thyroid hormones in maintaining cardiovascular homeostasis can be deduced from clinical and experimental data showing that even subtle changes in thyroid hormone concentrations adversely influence the cardiovascular system. Some potential mechanisms 
linking the two conditions are dyslipidaemia, endothelial dysfunction, blood pressure or body weight changes, and direct effects of thyroid hormones on the myocardium [4]. Moreover, thyroid hormones have an effect on glucose metabolism and probably on the development of insulin resistance (IR). In hyperthyroidism, impaired glucose tolerance may be the result of mainly hepatic IR, whereas in hypothyroidism the available data suggest that IR of peripheral tissues prevails [5]. However, the precise relationship between TDs, especially subclinical forms, and IR is still under discussion. IR represents a key pathogenetic mechanism of cardiometabolic diseases, and its prevention is crucial in the current dominance of cardiometabolic diseases in overall mortality [6, 7].

In addition to serum thyroid hormones, some studies have discussed the relationship between T3/T4 ratio and various, mostly metabolic, parameters or clinical outcomes [8,9]. The T3/T4 ratio is a calculated index used to indicate thyroid function and the action of hormones on the tissue. A method for detecting IR that is easy to use in common clinical practice is the homeostasis model assessment of IR (HOMA-IR). Its satisfactory correlation with the most accurate glucose clamp techniques has been confirmed by numerous studies. Even fasting insulin levels alone reflect IR to some extent [10].

The study aimed to investigate the association of IR with defined TDs and thyroid function parameters, and through these findings, identify such thyroid disorders and parameters that are the highest risk for the development of IR.

\section{Material and methods}

\section{Study population}

The study comprised 1425 middle-aged individuals (317 males, 1108 females) examined in a co-author's internal medicine outpatient centre in Hradec Králové, Czech Republic. Data were collected in 2009-2016 from the patients' first visits to the centre. The laboratory analyses below, including standard $75 \mathrm{~g}$ oral glucose tolerance test (OGTT), were performed, and, at the same time, their basic anthropometric parameters (waist circumference [WC], weight, and height) were measured. The obtained data were used to calculate body mass index (BMI) and HOMA-IR for each participant. Based on their TSH and FT4 levels and the manufacturer's instructions, all subjects were divided into subgroups according to Table 1 . None of the included subjects was treated with oral antidiabetic drugs or

Table 1. Study subgroups according to thyroid hormone levels

\begin{tabular}{lccc}
\hline Subgroups/thyroid status & TSH [mIU/L] & & FT4 [pmol/L] \\
\hline Euthyroidism & $0.4-4.0$ & and & $9-23$ \\
\hline Subclinical hypothyroidism & $>4.0$ & and & $9-23$ \\
\hline Overt hypothyroidism & $>4.0$ & and & $<9$ \\
\hline Hyperthyroidism & $<0.4$ & or & $>23$ \\
\hline
\end{tabular}

$\mathrm{TSH}$ — thyroid-stimulating hormone; FT4 — free thyroxine insulin. None of them had a history of thyroid disorders, and they were not treated with any drugs known to affect thyroid function. The study was conducted according to the principles stated in the Declaration of Helsinki. The study was approved by the Ethics Committee of University Hospital Olomouc and Faculty of Medicine and Dentistry, Palacký University Olomouc (Approval No. 20/11). To be included in the study, all subjects signed informed consent forms.

\section{Laboratory analysis}

Venous blood samples were drawn in the morning after a 12-hour fast. After centrifugation, the serum was used for analyses on the day of blood collection. Glucose was analysed on a Cobas 8000 (Roche Diagnostics $\mathrm{GmbH}$, Manheim, Germany) using the hexokinase method. Also on the Cobas 8000, TSH, FT4, and FT3 were measured by electrochemiluminescence immunoassay. Insulin was determined by chemiluminescent microparticle immunoassay on Architect i1000SR (Abbott Laboratories, Chicago, IL, USA). All analyses were performed according to the manufacturer's instructions and after verification of methods.

\section{Statistical analysis}

Statistical analysis was performed using the computing environment R (R Foundation for Statistical Computing, Vienna, Austria; http://www.r-project.org/). All numerical variables were characterised with descriptive statistics. The Wilcoxon signed-rank test (p-value) was used to compare numerical characteristics between the given subgroups. Extreme values of variables were found and excluded using the inner and outer fences method. All variables followed normal or near-normal distribution. Correlations of selected variables were quantified with Pearson's correlation coefficient (r), and the level of significance (p) was determined. Multivariate linear regression was performed to examine the relationships between thyroid hormones, age, and sex, as regressors of the dependent variables insulin and HOMA-IR. Due to a hyperbolic dependence of IR through the whole spectrum of TDs, evidenced by studies [5], the correlation and linear regression analyses were performed only in hypothyroid (both overt and subclinical) and euthyroid subjects. In other words, IR grows in both hypo- and hyper-thyroidism (in comparison with normal IR state in euthyroidism), whereas thyroid parameters change linearly through the whole spectrum. Hyperthyroidism was chosen for exclusion because of the expected lower prevalence in the study population. P-value of less than 0.05 indicates statistical significance. The following formula was used to calculates HOMA-IR (glucose levels in mmol/L, insulin levels in mIU/L) [10]:

HOMA-IR $=($ glucose $\times$ insulin $) / 22.5$

\section{Results}

\section{Characteristics of the study population}

The basic characteristics of all subjects with respect to a particular TD are shown in Table 2 . Only 23 individuals from the whole group met the criteria for hyperthyroidism; therefore, due to the disproportion to the size of the other subgroups, we did not include them into the table. As expected, most individuals had normal thyroid parameters, followed by the $\mathrm{OH}$ and $\mathrm{SH}$ subgroups, respectively. Between the subgroups, differences of the tested parameters, apart from the thyroid parameters, did not reach statistical significance. There were no apparent trends of the parameters between subgroups except for insulin, HOMA-IR, and 
Table 2. Basic characteristics in study subgroups - mean value (95\% confidence interval of the mean value)

\begin{tabular}{|c|c|c|c|c|c|}
\hline Characteristics & Euthyroidism & $\mathrm{p}$ value & $\begin{array}{c}\text { Subclinical } \\
\text { hypothyroidism }\end{array}$ & $\mathrm{p}$ value & Overt hypothyroidism \\
\hline \multirow{2}{*}{$\mathrm{N}$ (males, females) } & 1036 & \multirow[b]{2}{*}{-} & 76 & \multirow[b]{2}{*}{-} & 290 \\
\hline & $(243,793)$ & & $(11,65)$ & & $(57,233)$ \\
\hline \multirow{2}{*}{ Age [yrs] } & 42.21 & \multirow{2}{*}{0.397} & 43.94 & \multirow{2}{*}{0.452} & 43.23 \\
\hline & $(41.61 ; 42.81)$ & & $(43.21 ; 44.67)$ & & $(42.72 ; 43.75)$ \\
\hline \multirow{2}{*}{ BMI $\left[\mathrm{kg} / \mathrm{m}^{2}\right]$} & 31.17 & \multirow{2}{*}{0.204} & 32.98 & \multirow{2}{*}{0.172} & 32.04 \\
\hline & $(29.64 ; 32.79)$ & & $(25.66 ; 42.40)$ & & $(30.19 ; 34.00)$ \\
\hline \multirow{2}{*}{ Waist circumference $[\mathrm{cm}]$} & 109.69 (107.28; & \multirow{2}{*}{0.656} & 108.22 & \multirow{2}{*}{0.899} & 110.78 \\
\hline & $112.10)$ & & $(102.01 ; 114.43)$ & & $(108.78 ; 112.78)$ \\
\hline \multirow{2}{*}{ Glucose $[\mathrm{mmol} / \mathrm{L}]$} & 5.62 & \multirow{2}{*}{0.223} & 5.73 & \multirow{2}{*}{0.372} & 5.63 \\
\hline & $(5.58 ; 5.66)$ & & $(5.56 ; 5.90)$ & & $(5.56 ; 5.70)$ \\
\hline \multirow{2}{*}{ Insulin [mlU/L] } & 6.70 & \multirow{2}{*}{0.35} & 7.12 & \multirow{2}{*}{0.483} & 7.85 \\
\hline & $(6.42 ; 6.98)$ & & $(5.93 ; 8.55)$ & & $(7.26 ; 8.48)$ \\
\hline \multirow{2}{*}{ 2-hour OGTT } & 6.26 & \multirow{2}{*}{0.973} & 6.27 & \multirow{2}{*}{0.097} & 6.60 \\
\hline & $(6.16 ; 6.35)$ & & $(5.87 ; 6.71)$ & & $(6.42 ; 6.80)$ \\
\hline \multirow{2}{*}{ HOMA-IR } & 1.68 & \multirow{2}{*}{0.224} & 1.82 & \multirow{2}{*}{0.804} & 1.96 \\
\hline & $(1.60 ; 1.75)$ & & $(1.50 ; 2.21)$ & & $(1.80 ; 2.13)$ \\
\hline \multirow{2}{*}{ TSH [mlU/L] } & 1.91 & \multirow{2}{*}{$<0.001$} & 5.32 & \multirow{2}{*}{0.091} & 6.44 \\
\hline & $(1.86 ; 1.96)$ & & $(5.04 ; 5.61)$ & & $(2.58 ; 4.31)$ \\
\hline \multirow{2}{*}{ FT4 [pmol/L] } & 10.81 & \multirow{2}{*}{0.373} & 10.68 & \multirow{2}{*}{$<0.001$} & 8.28 \\
\hline & $(10.73 ; 10.90)$ & & $(10.37 ; 10.99)$ & & $(8.20 ; 8.36)$ \\
\hline \multirow{2}{*}{ FT3 [pmol/L] } & 5.21 & \multirow{2}{*}{0.711} & 5.18 & \multirow{2}{*}{0.876} & 5.13 \\
\hline & $(5.17 ; 5.26)$ & & $(5.00 ; 5.37)$ & & $(5.05 ; 5.22)$ \\
\hline \multirow{2}{*}{ FT3/FT4 ratio } & 0.48 & 0557 & 0.48 & - & 0621061.0621 \\
\hline & $(0.48 ; 0.49)$ & 0.551 & $(0.46 ; 0.51)$ & $<0.001$ & $0.02(0.01,0.00)$ \\
\hline
\end{tabular}

BMI — body mass index; OGTT — oral glucose tolerance test; HOMA-IR — homeostasis model assessment of insulin resistance; TSH — thyroid-stimulating hormone; FT4 — free thyroxine; FT3 — free triiodothyronine

Table 3. Pearson's correlation coefficients ( $r$ ) of thyroid function parameters

\begin{tabular}{lcccc}
\hline Characteristics & TSH & FT4 & FT3 & FT3/FT4 \\
\hline BMI & 0.008 & 0.035 & -0.055 & $-0.068^{*}$ \\
\hline Waist circumference & 0.002 & 0.033 & 0.001 & 0.038 \\
\hline Glucose & 0.014 & $0.064^{*}$ & 0.018 & -0.029 \\
\hline Insulin & -0.015 & $-0.081^{*}$ & $0.205^{* *}$ & $0.206^{* *}$ \\
\hline 2-hour OGTT & 0.007 & -0.026 & -0.007 & 0.044 \\
\hline HOMA-IR & -0.015 & -0.050 & $0.181^{* *}$ & $0.165^{* *}$ \\
\hline
\end{tabular}

${ }^{*} \mathrm{p}<0.05,{ }^{* *} \mathrm{p}<0.001$; TSH — thyroid-stimulating hormone; FT4 — free thyroxine; FT3 — free triiodothyronine; BMI — body mass index; 0GTT — oral glucose tolerance test; HOMA-IR — homeostasis model assessment of insulin resistance

two-hour glucose levels, which showed steady growth from euthyroidism through $\mathrm{SH}$ to $\mathrm{OH}$.

\section{Correlation analysis}

The highest correlation coefficients of thyroid parameters were noted with insulin levels and HOMA-IR as seen from Table 3, namely correlations of insulin with the FT3/FT4 ratio $(\mathrm{r}=0.206)$ and FT3 $(\mathrm{r}=0.205)$, respectively. Although the correlations were weak, they reached high statistical significance $(p<0.001)$. There were also highly significant correlations of both FT3 and the FT3/FT4 ratio with HOMA-IR ( $r=0.181$ and $r=0.165$, respectively). Figure 1 depicting the correlations of the FT3/FT4 ratio suggests no difference be- 


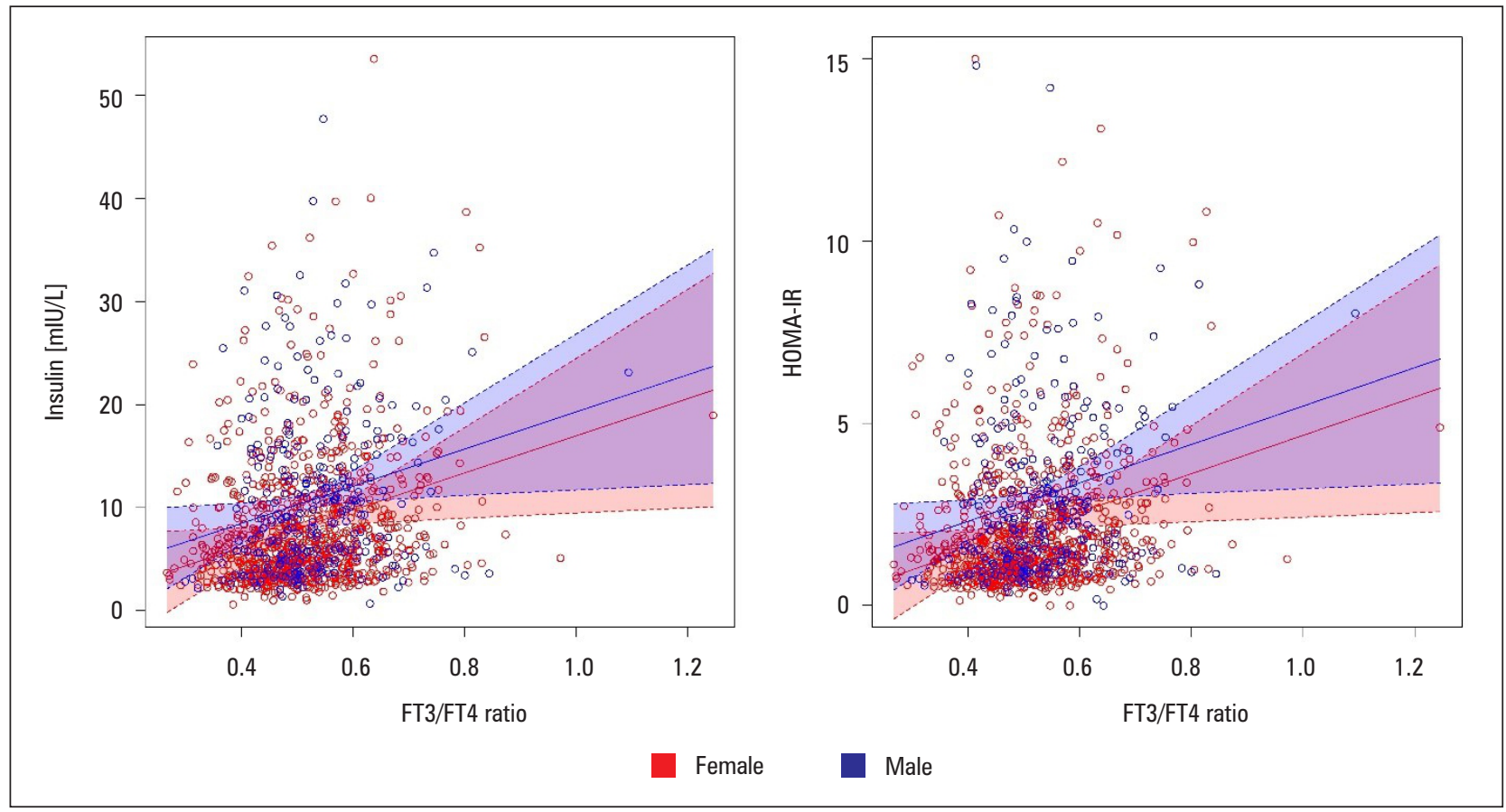

Figure 1. Scatter diagrams showing correlations of free triiodothyronine/free thyroxine (FT3/FT4) ratio with fasting insulin levels (A) and HOMA-IR (B) with 95\% confidence bands for the medians of variables on the $y$-axis for a given variable on the $x$-axis. There are no obvious sex differences in the relationship of the ratio and insulin resistance

Table 4. Effects of every 1-point increase of independent variables on the dependent variables HOMA-IR and insulin

\begin{tabular}{lccccccccc}
\hline \multirow{2}{*}{$\begin{array}{l}\text { Independent } \\
\text { variables }\end{array}$} & \multicolumn{2}{c}{ Linear coefficient } & \multicolumn{2}{c}{ Standard error } & \multicolumn{2}{c}{ t-value } & \multicolumn{2}{c}{ p-value } \\
\cline { 2 - 10 } & HOMA-IR & Insulin & HOMA-IR & Insulin & HOMA-IR & Insulin & \multirow{2}{*}{ HOMA-IR } & Insulin \\
\hline Intercept & -2.45 & -5.82 & 1.33 & 4.44 & -1.85 & -1.31 & 0.065 & 0.19 \\
\hline Age & 0.02 & 0.04 & $<0.01$ & 0.02 & 3.97 & 2.25 & $<0.001$ & 0.025 \\
\hline Sex (female) & -0.8 & -2.31 & 0.14 & 0.47 & -5.75 & -4.96 & $<0.001$ & $<0.001$ \\
\hline TSH & -0.01 & -0.05 & 0.02 & 0.05 & -0.75 & -0.96 & 0.455 & 0.338 \\
\hline FT4 & 0.16 & 0.44 & 0.12 & 0.39 & 1.39 & 1.12 & 0.166 & 0.261 \\
\hline FT3 & 0.04 & 0.23 & 0.25 & 0.84 & 0.17 & 0.27 & 0.862 & 0.785 \\
\hline FT3/FT4 ratio & 5.26 & 18.01 & 2.37 & 7.92 & 2.22 & 2.27 & 0.027 & 0.023 \\
\hline
\end{tabular}

HOMA-IR — homeostasis model assessment of insulin resistance; TSH — thyroid-stimulating hormone; FT4 — free thyroxine; FT3 — free triiodothyronine

tween males and females. As for the parameters not associated with thyroid functions, there were statistically highly significant correlations of WC and body weight with both insulin and HOMA-IR, the strongest being correlated body weight with HOMA-IR $(r=0.494)$.

\section{Regression analysis}

The impact of individual IR predictors is expressed with multivariate linear regression models that use linear coefficients to show the effects of one-point increases of these predictors (independent variables) on the dependent variables, HOMA-IR and insulin, respectively (Tab. 4). Except for the sex and age, the FT3/FT4 ratio was the only statistically significant predictor of both
HOMA-IR and insulin. If the ratio increases by one point, HOMA-IR and insulin will increase by a mean of $5.26(p=0.027)$ and $18.01(p=0.023)$, respectively. With each year of age, HOMA-IR and insulin will increase by a mean of $0.02(p<0.001)$ and $0.04(p=0.25)$, respectively. Female sex was a significant predictor of a lower insulin level and HOMA-IR in our study population.

\section{Discussion}

The obtained results of the correlation analysis revealed only a few statistically significant and very weak associations. The regression analysis confirmed a significant relationship only between IR and the FT3/FT4 ratio. The 
results also demonstrate both HOMA-IR and insulin levels increasing in the way from euthyroidism through $\mathrm{SH}$ to $\mathrm{OH}$, although without statistical significance. Epidemiological evidence indicates that both $\mathrm{OH}$ and $\mathrm{SH}$ are connected with IR. Some studies showed a statistically significant increase of HOMA-IR and insulin levels in subjects with SH compared to the euthyroid $[11,12]$. However, some findings showed no or insignificant difference between $\mathrm{SH}$ and the euthyroid state [13, 14]. A study by Wang et al. also recorded an increase in HOMA-IR between $\mathrm{SH}$ and $\mathrm{OH}$, as was the case in our study, although in our case without reaching statistical significance [12]. Comparable IR between $\mathrm{SH}$ and $\mathrm{OH}$ subjects was noted in a study by Maratou et al. [15]. We also demonstrate an increase of two-hour OGTT values in $\mathrm{SH}$ and $\mathrm{OH}$ compared to euthyroidism.

According to the population prevalence of TDs indicated in the introduction, hypothyroidism predominates. $\mathrm{SH}$ was estimated to affect up to $10 \%$, with the highest prevalence among women and elderly individuals. However, SH frequently reverts to euthyroidism, and TSH levels rise as people without TDs age, making it likely that the prevalence of $\mathrm{SH}$ may be overestimated [2]. The occurrence of TDs in our study sample does not exactly correspond to the proposed population prevalence. However, the study did not comprise a random adult population, but rather patients of a stated outpatient centre.

The most common cause of hypothyroidism in iodine-sufficient populations is Hashimoto's thyroiditis (HT). A chronic inflammation resulting from an imbalance between pro-inflammatory and anti-inflammatory cytokines in HT might be responsible for IR in hypothyroidism. Subjects with highly positive thyroid peroxidase antibodies (TPOabs) had elevated fasting insulin levels compared to those with lower titers of TPOabs, in a study by Mazarehi et al. [16]. OH was associated with obesity, promoting the production of pro-inflammatory cytokines and contributing to the development of IR. Moreover, BMI was positively correlated with TPOabs in men, suggesting that the pathogenesis of IR in hypothyroidism is a complex and interrelated process [17, 18]. In the present study, subjects with both $\mathrm{SH}$ and $\mathrm{OH}$ showed higher BMI values compared to euthyroidism.

Elevated levels of the FT3/FT4 ratio were associated with metabolic syndrome and some of its components such as obesity, elevated triglycerides, fasting blood glucose levels, and lower high-density lipoprotein cholesterol levels in euthyroid subjects [19, 20]. Based on regression analysis in a sample of 132,346 adults, Park et al. confirmed the FT3/FT4 ratio to have a better predictive power for metabolic syndrome than TSH [21]. In hypothyroidism and euthyroidism, as TSH levels rise the FT3/FT4 ratio also rises. Levothyroxine therapy was proved to decrease the FT3/FT4 ratio [22, 23]. Given the association of both hypothyroidism and the FT3/FT4 ratio with IR, thyroxine supplementation in indicated cases may help not only in achieving euthyroidism but also in improving IR.

The study has certain limitations. We considered all hypothyroid subjects as having a peripheral form (not distinguishing between central and peripheral forms) of hypothyroidism. However, due to the low prevalence of central hypothyroidism, there should be no major bias for this reason.

\section{Conclusion}

Both $\mathrm{SH}$ and $\mathrm{OH}$ are associated with increased HOMA-IR, fasting insulin levels, and two-hour OGTT glucose levels compared to euthyroidism. From the common laboratory parameters of the thyroid gland, the FT3/FT4 ratio shows the strongest relationship to IR. The FT3/FT4 ratio should be more emphasised in the diagnosis and treatment of TDs. After further verification, pharmacological lowering of the ratio could be a possible targeted way to reverse the development of IR. The results are valid for middle-aged European adults.

\section{Conflict of onterest}

Nothing to declare.

\section{Funding}

The study was supported by the grant IGA_LF_UPOL 2020_031.

\section{References}

1. Chaker L, Bianco A, Jonklaas J, et al. Hypothyroidism. Lancet. 2017; 390(10101): 1550-1562, doi: 10.1016/s0140-6736(17)30703-1, indexed in Pubmed: 28336049.

2. Biondi B, Cappola AR, Cooper DS. Subclinical Hypothyroidism: A Review. JAMA. 2019; 322(2): 153-160, doi: 10.1001/jama.2019.9052, indexed in Pubmed: 31287527.

3. Leo SDe, Lee S, Braverman L. Hyperthyroidism. Lancet. 2016; 388(10047): 906-918, doi: 10.1016/s0140-6736(16)00278-6, indexed in Pubmed: 27038492.

4. Jabbar A, Pingitore A, Pearce SHS, et al. Thyroid hormones and cardiovascular disease. Nat Rev Cardiol. 2017; 14(1): 39-55, doi: 10.1038/nrcardio.2016.174, indexed in Pubmed: 27811932.

5. Gierach M, Gierach J, Junik R. Insulin resistance and thyroid disorders. Endokrynol Pol. 2014; 65: 70-76, doi: 10.5603/EP.2014.0010, indexed in Pubmed: 24549605.

6. Garduño-Garcia Jd, Camarillo Romero E, Loe Ochoa A, et al. Thyroid function is associated with insulin resistance markers in healthy adolescents with risk factors to develop diabetes. Diabetol Metab Syndr. 2015; 7: 16, doi: 10.1186/s13098-015-0011-x, indexed in Pubmed: 25780389.

7. Samuel VT, Shulman GI. The pathogenesis of insulin resistance: integrating signaling pathways and substrate flux. J Clin Invest. 2016; 126(1): 12-22, doi: 10.1172/JCI77812, indexed in Pubmed: 26727229.

8. Emokpae MA, Obazelu PA. The Association of Triiodothyronine-to-Thyroxine Ratio with Body Mass Index in Obese Nigerian Children and Adolescents. Med Sci (Basel). 2017; 5(4), doi: 10.3390/medsci5040036, indexed in Pubmed: 29244733.

9. Pasqualetti G, Schirripa M, Dochy E, et al. Thyroid hormones ratio is a major prognostic marker in advanced metastatic colorectal cancer: Results from the phase III randomised CORRECT trial. Eur J Cancer. 2020; 133: 66-73, doi: 10.1016/j.ejca.2020.04.023, indexed in Pubmed: 32446145 . 
10. Wallace TM, Levy JC, Matthews DR. Use and abuse of HOMA modeling. Diabetes Care. 2004; 27(6): 1487-1495, doi: 10.2337/diacare.27.6.1487, indexed in Pubmed: 15161807.

11. Ebrahimpour A, Vaghari-Tabari M, Qujeq D, et al. Direct correlation between serum homocysteine level and insulin resistance index in patients with subclinical hypothyroidism: Does subclinical hypothyroidism increase the risk of diabetes and cardio vascular disease together? Diabetes Metab Syndr. 2018; 12(6): 863-867, doi: 10.1016/j.dsx.2018.05.002, indexed in Pubmed: 29748035.

12. Wang G, Liu J, Yang N, et al. Levothyroxine treatment restored the decreased circulating fibroblast growth factor 21 levels in patients with hypothyroidism. Eur J Intern Med. 2016; 31: 94-98, doi: 10.1016/j. ejim.2016.03.028, indexed in Pubmed: 27085392.

13. Maleki N, Kazerouni F, Hedayati M, et al. Assessment of cardiovascular risk factors in patients with subclinical hypothyroidism. Acta Cardiol. 2016; 71(6): 691-697, doi: 10.2143/AC.71.6.3178188, indexed in Pubmed: 27920457.

14. Aksoy N, Yeler MT, Ayan NN, et al. Association between thyroid hormone levels and insulin resistance and body mass index. Pak J Med Sci. 2015; 31(6): 1417-1420, doi: 10.12669/pjms.316.7560, indexed in Pubmed: 26870107.

15. Maratou E, Hadjidakis DJ, Kollias A, et al. Studies of insulin resistance in patients with clinical and subclinical hypothyroidism. Eur J Endocrinol. 2009; 160(5): 785-790, doi: 10.1530/EJE-08-0797, indexed in Pubmed: 19141606.

16. Mazaheri T, Sharifi F, Kamali K. Insulin resistance in hypothyroid patients under Levothyroxine therapy: a comparison between those with and without thyroid autoimmunity. J Diabetes Metab Disord. 2014; 13(1): 103, doi: 10.1186/s40200-014-0103-4, indexed in Pubmed: 25364704.
17. Sanyal D, Raychaudhuri M. Hypothyroidism and obesity: An intriguing link. Indian J Endocrinol Metab. 2016; 20(4): 554-557, doi: 10.4103/2230-8210.183454, indexed in Pubmed: 27366725.

18. Wang B, Song R, He W, et al. Sex Differences in the Associations of Obesity With Hypothyroidism and Thyroid Autoimmunity Among Chinese Adults. Front Physiol. 2018; 9: 1397, doi: 10.3389/fphys.2018.01397 indexed in Pubmed: 30337885

19. Xu R, Huang F, Zhang S, et al. Thyroid function, body mass index and metabolic risk markers in euthyroid adults: a cohort study. BMC Endocr Disord. 2019; 19(1): 58, doi: 10.1186/s12902-019-0383-2, indexed in Pubmed: 31174521.

20. Urrunaga-Pastor D, Guarnizo-Poma M, Moncada-Mapelli E, et al. Insulin Resistance and Metabolic Syndrome Research Group. High free triiodothyronine and free-triiodothyronine-to-free-thyroxine ratio levels are associated with metabolic syndrome in a euthyroid population. Diabetes Metab Syndr. 2018; 12(2): 155-161, doi: 10.1016/j.dsx.2017.12.003, indexed in Pubmed: 29254889.

21. Park SoY, Park SeE, Jung SW, et al. Free triiodothyronine/free thyroxine ratio rather than thyrotropin is more associated with metabolic parameters in healthy euthyroid adult subjects. Clin Endocrinol (Oxf). 2017 87(1): 87-96, doi: 10.1111/cen.13345, indexed in Pubmed: 28374508.

22. Ettleson MD, Bianco AC. Individualized Therapy for Hypothyroidism: Is T4 Enough for Everyone? J Clin Endocrinol Metab. 2020; 105(9), doi: 10.1210/clinem/dgaa430, indexed in Pubmed: 32614450.

23. Strich D, Chay C, Karavani G, et al. Levothyroxine Therapy Achieves Physiological FT3/FT4 Ratios at Higher than Normal TSH Levels: A Novel Justification for T3 Supplementation? Horm Metab Res. 2018; 50(11): 827-831, doi: 10.1055/a-0751-0498, indexed in Pubmed: 30396211. 